\section{DARC: a new method for detecting progressive neuronal death}

\begin{abstract}
Visual field evaluations are currently used as the gold standard in assessing disease progression in glaucoma, but this method considers damage that has irreversibly occurred. If retinal ganglion cell (RGC) apoptosis can be detected, it may be possible to prevent loss of vision, and also to act at a much earlier stage to avoid the secondary degeneration of neurons and progression of the disease. Detection of apoptosing retinal cells (DARC) is a method that uses advanced optical techniques to image and track the process of RGCs in vivo over time. It has the potential to help elucidate the cellular mechanisms involved in the development of RGC degeneration and vision loss in glaucoma. Eye (2007) 21, S15-S17; doi:10.1038/sj.eye.6702881
\end{abstract}

Keywords: Glaucoma; retinal ganglion cells; apoptosis; in vivo imaging

\section{Introduction}

Currently, it is not possible to assess accurately the extent of neuronal damage in glaucoma. Visual field measurement is the 'gold standard' for such an assessment, but this approach provides an indication of damage that has already occurred and does not give information on the apoptosis of retinal ganglion cells (RGCs), which marks the initiation of the disease process. If glaucomatous change at this level can be detected, it may be possible to initiate treatment earlier, avoid the secondary degeneration of neurons, and prevent progression of the disease and irreversible vision loss.

\section{Detection of apoptosing retinal cells}

\section{Animal findings}

Dr Cordeiro's research team has produced the first video of single neuronal cells undergoing

MF Cordeiro

apoptosis in a living eye, a rat eye in which apoptosis was induced using staurosporine. Figure 1 shows a retinal montage of a rat eye. Individual RGCs undergoing apoptosis can be seen at what is believed to be one of the earliest stages in glaucomatous damage. The technique used, termed detection of apoptosing retinal cells (DARC), depends on a fluorescent signal being detected by a scanning laser ophthalmolscope. ${ }^{1}$

This in vivo demonstration of RGC apoptosis was preceded with histological proof of concept. RGCs of a rat model were labelled with DiAsp through retrograde labelling to the superior colliculus. Apoptosis was detected using apoptosis markers. Every normal cell contains phosphatidylserine molecules, and during apoptosis, these are translocated to the outside of the membrane. Annexin is a molecule that has a high affinity for these exposed phosphatidylserine molecules, providing a positive label for apoptotic cells, which were co-localized to the labelled RGC. A second apoptosis marker, anti-caspase-3, was used to confirm that annexin was truly acting as a marker for apoptosis in the model.

Using the Morrison rat model of glaucoma (in which the intraocular pressure of one eye was elevated by injection of hypertonic saline solution into the episcleral veins), a dose-dependent response to the integral intraocular pressure (IOP) was found. This was also found when eyes were treated with staurosporine - a potent inducer of neuronal apoptosis - such that at low doses, fewer cells became apoptotic than at a higher dose. At the sites of RGC apoptosis in the rat ocular hypertension $(\mathrm{OHT})$ model, there was increased expression of the matrix metalloprotease MMP-9. The researchers showed that the extracellular matrix component to which the RGC adhered was laminin, and that there was
UCL Institute of Ophthalmology and Western Eye Hospital, London, UK

Correspondence: MF Cordeiro, UCL Institute of Ophthalmology and Western Eye Hospital, London, UK Tel: +442076086938. E-mail: M.Cordeiro@ ucl.ac.uk 


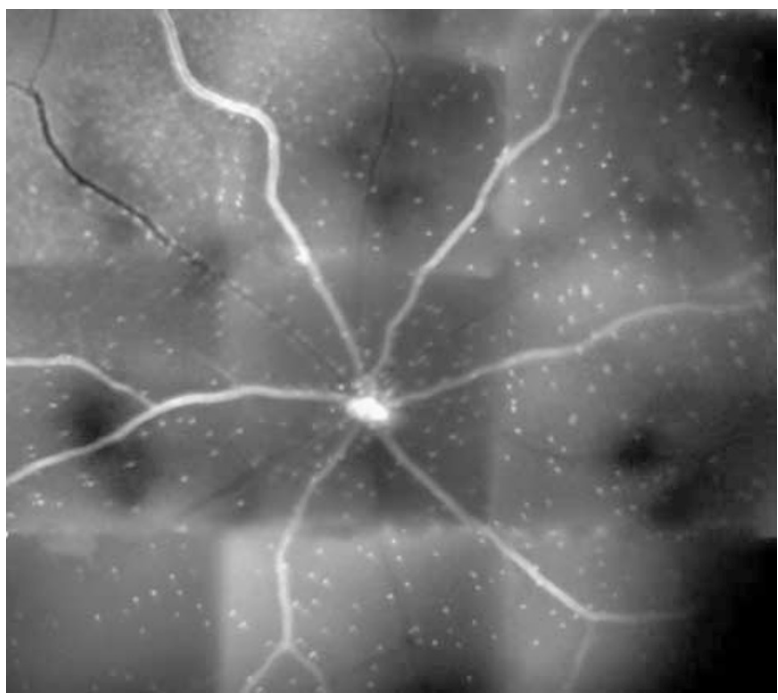

Figure 1 In vivo apoptosis of neuronal cells in a rat eye; each white dot represents an individual RGC undergoing apoptosis, and different cells are lighting up (turning white) at different times.

significantly reduced expression of laminin in eyes that had apoptosing RGCs. ${ }^{2}$ They moved on to demonstrate that RGC apoptosis in vivo in the same part of the retina, using the same OHT model eye, could be followed longitudinally over time. A large number of RGCs were lost by 8 weeks after IOP increase had been induced in the animal eye; apoptosis peaked 3 weeks after elevated IOP had been induced.

DARC has also been used in the primate eye, comparing a baseline picture and a picture taken after RGC apoptosis was induced with staurosporine. The individual RGCs were clearly visible and it was easy to distinguish those that were dying. ${ }^{1}$

\section{Potential clinical applications}

The next stage for DARC would be its application to the clinic. For patients, DARC could be a tool to monitor both glaucoma progression and treatment outcomes. The use of DARC in assessing potential antiglaucoma strategies is probably the most immediate promising application, with the technique having already been evaluated in this way using animal models. ${ }^{3}$ Using several glutamate modulators including MK801, which is an N-methyl-D-aspartate antagonist very similar to memantine, they showed a dose-dependent effect on RGC apoptosis. Eyes were imaged in vivo using DARC, and the results were confirmed histologically (Figure 2). In a rat OHT model, when IOP was increased over 3 weeks, the level of RGC apoptosis differed depending on the time

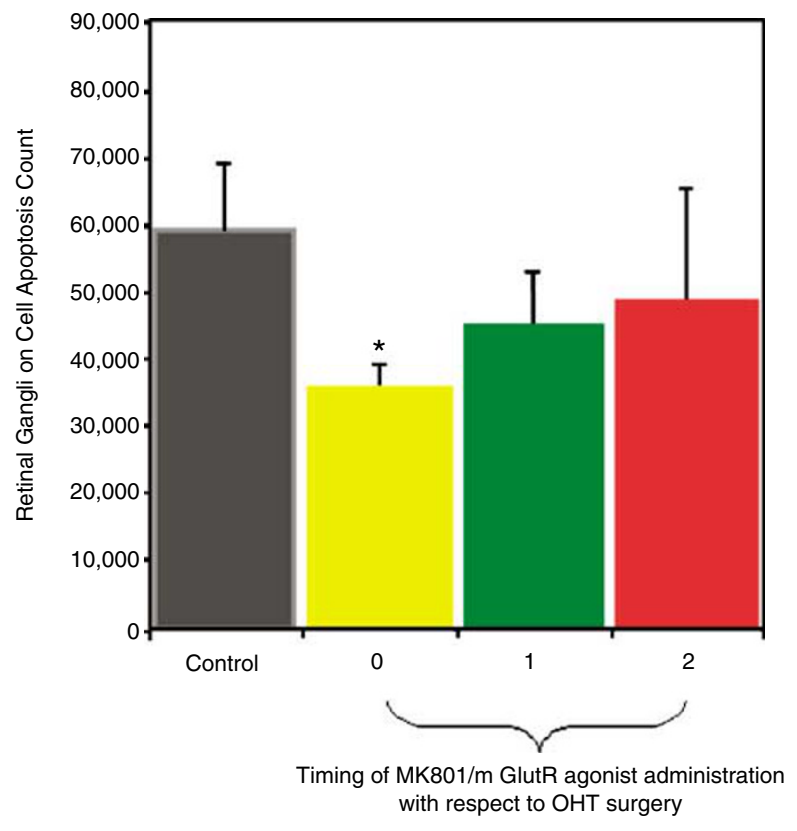

Figure 2 Using DARC, the effect of different times of administration (in weeks) of glutamate modulators on RGC apoptosis in a rat model of OHT were assessed. Maximal rescue was acheived when treatment was given at the time of surgical induction of elevated IOP. Asterisk indicates significantly different from control.

at which the glutamate agent was applied - when IOP was raised, 1 week or 2 weeks after IOP was raised. The most significant effect (ie, depression of apoptosis) occurred when the treatment was given at the time IOP was raised, suggesting the involvement of secondary degeneration.

\section{Conclusion}

Conventional clinical tests detect glaucoma but not until irreversible neuronal damage has occurred. DARC provides a way of imaging this damage at an earlier stage in the disease process - at the point of neuronal apoptosis. This approach can help to unveil some of the mechanisms involved in the development of apoptosis and vision loss in glaucoma, and also to gauge when to start treatment to prevent VF loss. In addition, monitoring treatment effectiveness would also be possible. DARC is being refined for its application to patients.

\section{References}

1 Cordeiro MF, Guo L, Luong V, Harding G, Wang W, Jones HE et al. Real-time imaging of single nerve cell apoptosis in retinal neurodegeneration. Proc Natl Acad Sci USA 2004; 101(36): 13352-13356. 
2 Guo L, Moss SE, Alexander RA, Ali RR, Fitzke FW, Cordeiro MF. Retinal ganglion cell apoptosis in glaucoma is related to intraocular pressure and IOP-induced effects on extracellular matrix. Invest Ophthalmol Vis Sci 2005; 46(1): 175-182.
3 Guo L, Salt TE, Maass A, Luong V, Moss SE, Fitzke FW et al. Assessment of neuroprotective effects of glutamate modulation on glaucoma-related retinal ganglion cell apoptosis in vivo. Invest Ophthalmol Vis Sci 2006; 47(2): 626-633. 